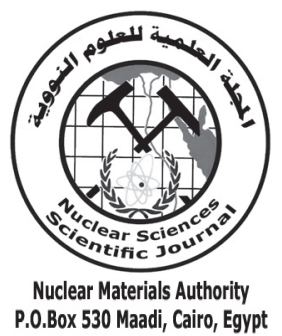

ISSN 2314-5609

Nuclear Sciences Scientific Journal

vol. 2, p 133 - 137

2013

\title{
VEHICLE DETECTION FROM REMOTE SENSING IMAGES
}

\author{
IRAKY H. KHALIFA and ABDUALRHMAN A. ALZIDAN
}

Fac. Computers and Information, Helwan Univ.

\begin{abstract}
Image object detection is an important application of remote sensing technology. Road vehicle detection using very high-resolution remote sensing images has a unique advantage of covering a large area such as jaddah and alriad at the same time over all ground-based detectors. But the detection of small vehicle-object in remote sensing imagery is still a challenging task. Several parameters of gray values and sizes are examined to classify the objects in the image. The vehicles and their associated shadows can be discriminated by removing big objects such as roads. Our test shows a promising result of detecting the vehicle. We present an object-based detection approach with IMAGINE Objective for the detected vehicles in the study area. (jeddah city, alriad city )

IMAGINE Objective employs feature models working on objects produced by image segmentation and various other pixel-based algorithms can be processed by geometric and textural parameters after being vectorised. This process is used and optimised for the extraction of the vehicles from high resolution images. Preliminary object detection tests using a semi automated post-classification approach show reliable results. For this research, the applied methods prove to be useful to detect vehicles on the road of example images even without producing a complete extraction of all vehicles. The approach will be extended to the whole images area.

\section{INTRODUCTION}

With the availability of sub-metric resolution remotely sensed imagery, the automatic detection, characterization and monitoring of traffic vehicles using (airborne or satellite image) with its unique wide-area coverage and synoptic view has become an emerging field of research ( Hinz et al.,2006 ; Gerhardinger et al.,2005 and Jin \&Davis ,2007). Currently, more reliable vehicle detection can only be achieved from the very high-resolution remote sensing images. Existing approaches for vehicle detection can be categorized based on the underlying type of vehicle modeling (Shrma et al.,2006; Schlosser et al.,2003; Stilla et al.,2004; Hinz et al.,2005). There are two basic kinds of vehicle models that have been used: (1) an appearance-based implicit model by clustering appropriate pixel groups into potential

vehicles. The implicit model typically consists of intensity or texture features surrounding each pixel. Detection is performed by checking feature vectors surrounding image pixels. (2) an explicit model. An explicit model usually describes a vehicle as a box or wire-frame representation. Matching the model top-down to the image, or grouping extracted image features bottom-up to create structures similar to the model is performed on the image. We present an object-based detection approach with Imagine Objective (Erdas,2010)for detect vehicles in the project area. Imagine $\mathrm{Ob}-$ jective employs feature models which work on objects produced by image segmentation and various other pixel-based algorithms can be processed by geometric and textural parameters after being vectorised. This process is used and optimized for the extraction of the vehicles from high resolution images.
\end{abstract}




\section{Data and Methodology \\ Data}

The Available data of the study area of roads are two Aerial photo( Panchromatic image, res. $60 \mathrm{~cm}$ and Pan Aerial photo res. $15.24 \mathrm{~cm}$ ) and satellite image ( Worldview2 images, 4bands (2Red; 3Green,5 Blue, and Infrared of $2 \mathrm{~m}$ resolution) (Fig. 1).

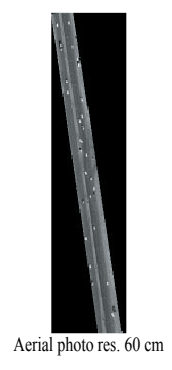

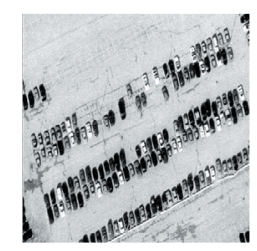

Aerial photo res. $15.24 \mathrm{~cm}$

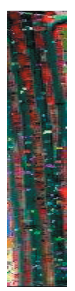

satellite Worldview2 image (4bands, 2 Red 3 Green,5 Blue, and Infrared; res. $2 \mathrm{~m}$
Fig.1: Original Images

\section{Methods}

Image processing was conducted in ERDAS Imagine 10, using the Imagine Objective tool (Erdas,2010) which enables automated feature extraction. An object based classification was used to identify vehicles in the Aerial photo and satellite image. Object based classification is defined as assigning classes to image objects which are the result of segmentation of an image into discrete non-overlapping units based on specific criteria. The object based classification consisted of five processes: probability matrix computation, image segmentation, raster to vector conversion, vector processing, and vector cleanup. The probability matrix computation is a supervised procedure being trained using the location of known vehicle. The probability matrix computation assigns a probability of being a vehicle or not to each pixel. The probability of being a vehicle or not is determined based on the similarity of spectral values of the given pixel to the spectral values of the pixels of known vehicle identified during the training process. Segmentation of an image is the process of partitioning a digital image into multiple regions to simplify and/or change the representation of an image into something that is more meaningful and easier to analyze. The segmentation based on spectral values and spatial characteristics created objects that are useful in classifying land cover in regions. The segmentation process was performed on the original Aerial photo and 4 band satellite images; however, the pixel probability layer was also used to calculate the probability zonal mean for each generated segment. The segmentation approach used combined splitting and merging of the input image with the use of edge detection to identify segments with similar characteristics. Threshold and Clump approach use to get all possible vehicle pixels to generate vehicle raster objects.

The parameters used for (Aerial photo, resolution. $15 \mathrm{~cm}$ ) image segmentation included: Euclidean distance (the compute settings function was used to determine the minimum value difference and the variation factor, 40 and 3.5 respectively), edge detection with an automatically generate threshold of 53, and a minimal edge length of three pixels.

The parameter used for (Aerial photo ,resolution. $60 \mathrm{~cm}$ and satellite worldview2, 4band, res. $2 \mathrm{~m}$ ) images Threshold and Clump included: Probability threshold $=0.50$.The outputs images from segmentation of (Aerial photo) and Threshold and Clump (Aerial photo) image res 60 and satellite image (4 bands) res. $2 \mathrm{~m}$ were then converted into a vector files, which was used for the vector object processing and cleanup procedures. Vector object processing consists of another supervised classification which utilizes geometry to refine the identification of vehicles. A variety of geometric cues are available for use in vector object processing, however after experimenting with the cue options we selected the following cues for use: area, a perimeterarea ratio, and shadow. The thresholds used for the cues were determined by training the classification with the area and perimeter-area 
characteristics of the vehicle observed on the road. The area characteristic had a minimum value of 4 square meters and a maximum of 24 square meters. The shadow cue measures the association (determined based on adjacency) of the vector objects generated from the first three steps of the object based procedure to a shadow polygon created using a separate unsupervised classification. The vector cleanup process consisted of applying a probability filter to the vector file. The probability filter removed all object with less than 0.9 probability of being a vehicle, thereby ensuring that the final vector layer only included polygons that had a high certainty of being vehicle . An assessment of the accuracy of the object based classification was conducted comparing the location of vehicle polygons as identified in the final vector layer from the object based classification with the actual spatial location of individual vehicles as determined in the road( Fig. 2, 3, and 4).

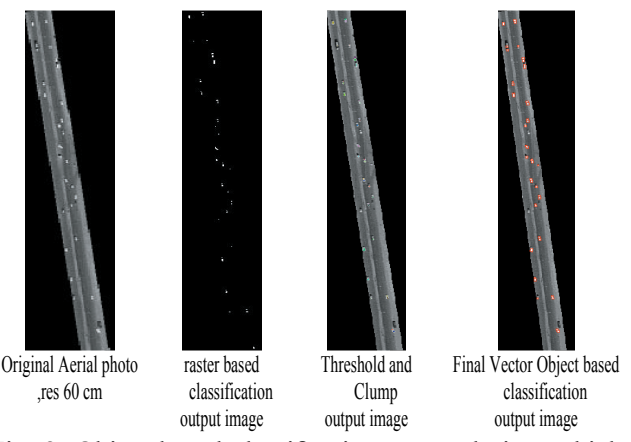

Fig. 2: Object based classification stages during vehicle detection from Aerial photo (res $60 \mathrm{~cm}$ )

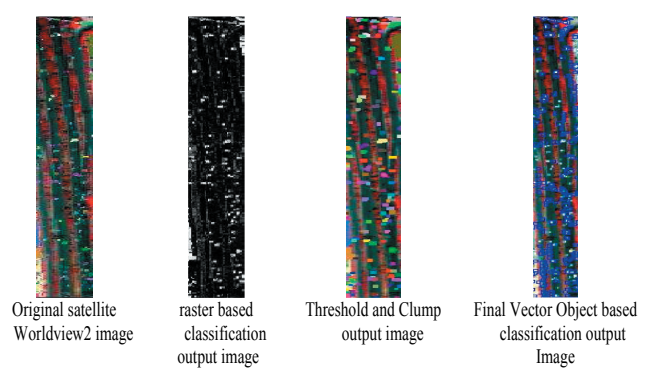

Fig. 3: Object based classification stages during ,Worldview2 satellite image (3band, res $2 \mathrm{~m}$ ) And Vector Object based classification output Image
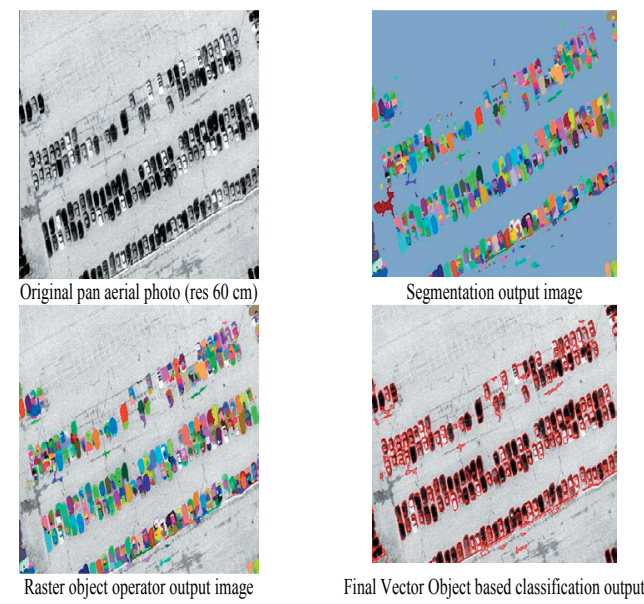

image

Fig. 4: Object based classification stages during vehicle detection from Aerial photo (res $15.24 \mathrm{~cm}$ )

\section{RESULTS AND DISCUSSION}

The object based classification results in a vector file containing polygons which are associated with one of two classes: class 1- vehicles, class 2-non vehicle. For the purpose of this study we are interested initializing object based classification to discriminate vehicles from the background. The object based classification improves upon a pixel based classification by incorporating shapes and spatial associations (e.g., association with shadow) of vehicles; additionally the resulting vector layer includes shape and size in the characterization of vehicles. The results from the object based classification were considered in terms of number of correctly identified vehicles, as determined by comparing the location of vehicles identified in the object based classification to the actual spatial locations of individual vehicles identified in the road. The results are compared with visual detection results (Table 1). There were 25 vehicles in Aerial photo (res $60 \mathrm{~cm}$ ), and 184 vehicles in Aerial photo (res $15.24 \mathrm{~cm})$, and 112 vehicles in Worldview image (4bands, res $2 \mathrm{~m}$ ) were extracted correctly by the process of vehicle detection. Only 3,1, and 0 vehicles respectively were missed, and the noises which are not vehicles but extracted as vehicles were 0,15 , and 52 , respectively 
. The producer accuracy is $89 \%, 99 \%$, and $100 \%$, respectively and the user accuracy is $89 \%, 92 \%$ and $69 \%$, respectively. In the images, 28, 169, and 112 vehicles, respectively were extracted correctly. Overall, almost all the vehicle could be extracted.

Table 1: Accuracy of vehicle detection

\begin{tabular}{|c|c|c|c|}
\hline Image & $\begin{array}{r}\text { Aerial } \\
\text { photo } \\
(\text { res } 60 \\
\text { cm) }\end{array}$ & $\begin{array}{l}\text { Aerial photo } \\
\text { (res } 15 \mathrm{~cm})\end{array}$ & $\begin{array}{c}\text { Worldview2 } \\
\text { image } \\
\text { (3bands, res 2m) }\end{array}$ \\
\hline Vehicle in image & 28 & 169 & 112 \\
\hline Extract result & 25 & 184 & 162 \\
\hline Correctly extracted & 25 & 168 & 112 \\
\hline 0mission & 3 & 1 & 0 \\
\hline Commission & 0 & 15 & 52 \\
\hline Produser Accuracy & 89 & 99 & 100 \\
\hline User accuracy & 89 & 92 & 69 \\
\hline
\end{tabular}

Not only light-color vehicles but also dark vehicles and some vehicles in shadow were extracted successfully. Because we extracted both vehicles and shadows, even the vehicle's gray value is similar to that of the road; the vehicle can be extracted by its associated shadow. There still have a few commission errors due to a Worldview2 image (4bands, res $2 \mathrm{~m}$ ), its shadow and some lines on the road. The environment condition around the target area influences the result of vehicle detection. Accuracy gets higher as an environment becomes simpler.

\section{CONCLUSION}

Object based classification of high resolution imagery provides the tools to detect vehicles in the road, object-based detection approach with Imagine Objective for the detect vehicles in the test images. Preliminary object detection tests using a semi automated postclassification approach show reliable results. For this research, the applied methods prove to be useful to detect vehicles in the road of example images even without producing a complete extraction of all vehicles in whole images. The approach will be extended to the whole images area.

\section{REFERENCES}

ERDAS, 2010 . Inc. IMAGINE Objective Users Guide (January, 2009). Norcross, GA

Gerhardinger, A.; Ehrlich, D., and Pesaresi, M., 2005. Vehicles detection from very high resolution satellite imagery. Inter. Archives of Photogrammetric and Remote Sensing, XXXVI, Part 3/W24, 83-88.

Hinz, S. ; Leitloff, J., and Stilla, U.,2005.Contextsupported vehicle detection in optical satellite images of urban areas. Proc. Inter. Geoscience and Remote Sensing Symposium, Seoul, Korea, 2937-2941.

Hinz, S.; Bamler, R., and Stilla, U., 2006. Theme Issue: Airborne and Spaceborne Traffic Monitoring. ISPRS J. Photogrammetric and Remote Sensing. Elsevier, 61,135-136.

Jin, X., and Curt H. Davis, 2007 .Vehicle detection from high resolution satellite imagery using morphological shared-weight neural networks. Image and Vision Computing, 25, 1422-1431.

Schlosser, C.; Reitberger, J., and Hinz, S. ,2003. Automatic car detection in high resolution urban scenes based on an adaptive 3D-model. Proc.2nd GRSS/ISPRS Joint Workshop on Data Fusion and Remote Sensing over Urban Area, Berlin, Germany, 167-170.

Sharma, G.; Merry, J. C.; Goel, P., and McCord, M., 2006. Vehicle detection in 1-m resolution satellite and airborne imagery. Inter. J. Remote Sensing, 27, No.4, 779-797.

Stilla, U. ; Michaelsen, E.; Soergel, U.; Hinz, S., and Ender, H.J.,2004. Airborne monitoring of vehicle activity in urban areas. Inter. Archives of Photogrammetry and Remote Sensing, 35, 973-979. 
الكثف عن المركبات بإستخدام تكنولوجيا الأستشعار عن بعد

عر اقي خليفه و عبد الرحمن الزيدان

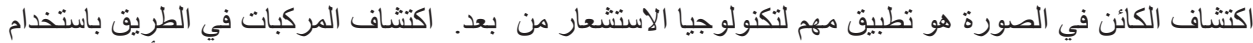

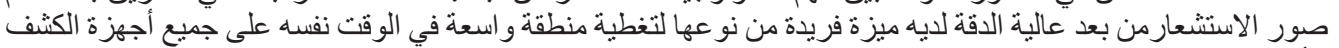

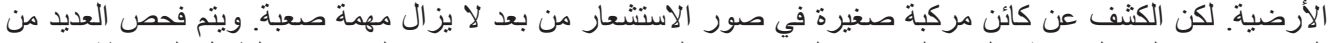

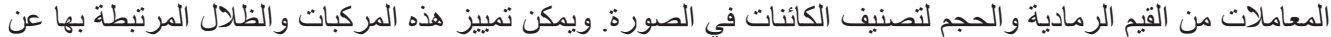

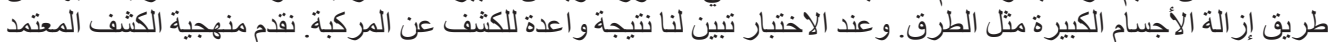

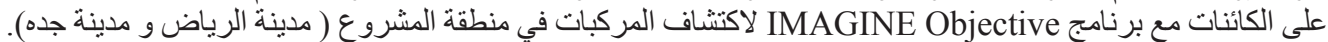

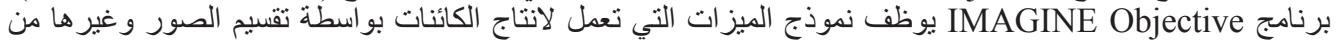

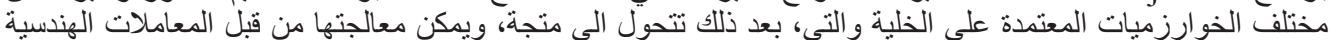

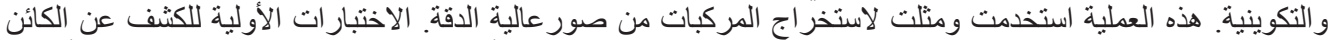

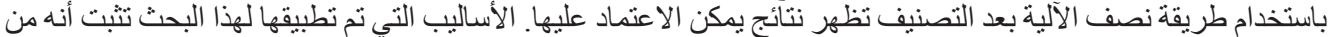

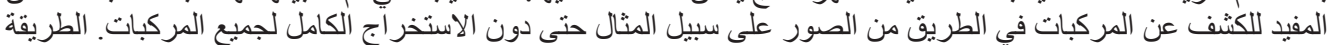
المتبعة سوف يتم توسيعها لتشمل نطاق لمنيق منطقة صورة كاملة. 